\title{
A new anesthesia scheme for parathyroidectomy under neuromonitoring: a retrospective cohort study
}

\author{
Danyang Wang, Linlin Li, Chunlu Zhang, Feng Liang, Peng Chen \\ Department of Anesthesiology, China-Japan Union Hospital of Jilin University, Changchun, China \\ Contributions: (I) Conception and design: D Wang, L Li; (II) Administrative support: P Chen; (III) Provision of study materials or patients: C Zhang, \\ F Liang; (IV) Collection and assembly of data: D Wang, L Li; (V) Data analysis and interpretation: D Wang, L Li; (VI) Manuscript writing: All \\ authors; (VII) Final approval of manuscript: All authors. \\ Correspondence to: Peng Chen. China-Japan Union Hospital of Jilin University, 126 Xiantai Dajie, Jingkai District, Changchun 130033, China. \\ Email: cpeng@jlu.edu.cn.
}

\begin{abstract}
Background Parathyroidectomy under nerve monitoring has been carried out for nearly ten years in the China-Japan Union Hospital of Jilin University. We retrospectively evaluated patients' prognosis with secondary hyperparathyroidism (SH) under neuro- and non-neuro-monitored parathyroidectomy anesthesia. The purpose of this study is to summarize and introduce a new anesthesia scheme for parathyroidectomy under nerve monitoring.

Methods: From January 2000 to December 2019, 200 patients with SH in the China-Japan Union Hospital of Jilin University were retrospectively analyzed. Among them, 100 patients underwent parathyroidectomy under neurological monitoring (Group A), and 100 patients underwent parathyroidectomy without neurological monitoring (Group B). The dosage of muscle relaxant, parathyroid hormone (PTH), serum calcium, phosphorus, urea, creatinine, and alkaline phosphatase (ALP) was recorded before surgery (T0), after surgery (T1), at discharge (T2), during skin incision (Ta), at four parathyroidectomies (Tb), and 10 min after total removal. The levels of PTH were measured at four-time points (Tc) and 30 minutes (Td) after complete resection.
\end{abstract}

Results: After screening and propensity score match (PSM), the data of 92 patients were analyzed. Group A's muscle relaxant dose was significantly less than Group B; the length of hospital stay in Group A was significantly lower than in Group B $(\mathrm{P}<0.05)$. The serum calcium levels, phosphorus, urea, and creatinine at $\mathrm{T} 2$ in Group A were lower than those in Group B $(\mathrm{P}<0.05)$.

Conclusions: Parathyroid nerve monitoring technology combined with preoperative complete anesthesia scheme, anesthesia induction with one time ED95 (95\% effective drug dose) cis-atracurium, end breath gas, and sevoflurane maintenance anesthesia under BIS monitoring can improve the prognosis of patients, shorten the length of hospital stay, and is effective and safe.

Keywords: Nerve monitoring; secondary hyperparathyroidism (SH); anesthesia scheme

Submitted Jan 17, 2021. Accepted for publication Mar 29, 2021.

doi: 10.21037 /gs-21-33

View this article at: http://dx.doi.org/10.21037/gs-21-33

\section{Introduction}

Most patients with chronic renal failure (CRF), especially in developing countries, develop into secondary hyperparathyroidism $(\mathrm{SH})$ if they do not receive advanced and appropriate professional treatment in the early stage of the disease (1-3). These patients had elevated serum phosphorus levels and decreased calcium levels. $\mathrm{SH}$ produces a group of syndromes with abnormal facial appearance, which was first summarized and named as Sagliker syndrome (SS) by Dr. Sagliker of Turkey in 2004 $(3,4)$. SS patients with short stature or shorter than before, 
hearing loss, clubbing fingers, osteoporosis is vulnerable. The face is ugly, and the maxilla grows forward and downward as a whole, and the anterior part of the mandible increases vertically. Finally, the patient's whole face is larger than that before the onset of the disease, and the front looks like a hippopotamus, which causes severe psychological problems. There are tumors and ulcers in the oral cavity, and the teeth present " $\mathrm{X}$ " or "O" irregular changes, which increase the difficulty of intubation (5-7).

Parathyroidectomy is the first choice for patients with $\mathrm{SH}$. Iatrogenic recurrent laryngeal nerve (RLN) injury is a common complication in parathyroid surgery (8). The abnormal rate of parathyroid anatomical position is high, and it is often closely related to the RLN. In recent years, intraoperative neural monitoring (IONM) is a revolutionary technology in China to reduce RLN injury during thyroid and parathyroid injury. The China-Japan Union Hospital of Jilin University's thyroid surgery department carried out this technology in 2010 (9).

IONM simulates nerve impulse with a pulse current, which causes muscle fiber to produce compound muscle action potential and record it. By analyzing the amplitude, latency, and duration of an action potential, the conduction function of motor nerve meridian can be judged, and visual electromyography (EMG) can be formed $(10,11)$. During the surgery, the direction of RLN and whether there is mechanical and energy damage during surgery can be judged by waveform and numerical value. At present, the domestic use of conventional visual identification, postoperative temporary, and permanent nerve injury, respectively, is $2.58 \%$ and $1.07 \%$; after using IONM, it is reduced to $1.82 \%$ and $0.67 \%$, respectively (12). The working principle of IONM is that the motor nerve is stimulated by electric current, and the action potential is formed to cause the muscle to contract, thus obtaining electromyography. The use of neuromuscular blocking agents interferes with the acquisition of standard nerve EMG signals, which requires the rational use of neuromuscular blocking agents in surgery. During anesthesia induction, muscle relaxant drugs were not used for intubation, although it was conducive to acquiring intraoperative nerve EMG signals.

However, the technical surgery is more complicated; the intubation response is massive, prone to an intubation side injury, and affects patients' hemodynamic stability (13). How to achieve a win-win situation between the surgeons and anesthesiologists poses a new challenge to anesthesia management. Through a series of studies, we put forward the concept of low-dose muscle relaxation induction for this kind of surgery. We recommend cis-atracurium as a muscle relaxant. Cis-atracurium is a non-depolarizing muscle relaxant, which Hofmann degrades without renal damage. It is the first choice of muscle relaxant in patients with renal failure and SH. Through clinical experiments, it is verified that one time of ED95 cis-atracurium is suitable for parathyroid surgery under nerve monitoring $(14,15)$. This dosage meets the muscle relaxation requirements of intubation and does not affect nerve EMG signals acquisition by specialists.

Besides, sevoflurane is recommended for the maintenance of anesthesia. Sevoflurane does not affect renal function and has an excellent central muscle relaxation effect, which makes up for the deficiency of a small dose of neuromuscular blocking agents (16). Also, sevoflurane inhalation anesthesia needs end-expiratory gas monitoring and BIS (bispectral index) anesthesia depth monitoring equipment, which can fine adjust the depth of anesthesia and significantly reduce agitation incidence. The advantage of total inhalation anesthesia is that the center of anesthesia is accurate and easy to adjust. The depth of anesthesia was maintained at 1.2-1.4 MAC. At this depth of anesthesia, it can meet anesthesia depth and analgesia requirements and produce a certain degree of central muscle relaxation. Anesthesia is relatively easy to maintain and does not affect the acquisition of nerve EMG signals. Therefore, standardized anesthesia management can ensure the smooth development of parathyroid surgery under nerve monitoring.

We present the following article in accordance with the STROBE reporting checklist (available at http://dx.doi. org/10.21037/gs-21-33).

\section{Methods}

\section{Patients}

The study was approved by the Ethics Committee of ChinaJapan Union Hospital of Jilin University (No. 2021-KYLL030002). The patients have given informed consent. The study was conducted in accordance with the Declaration of Helsinki (as revised in 2013).

In 2010, the intraoperative monitoring technology of RLN in thyroid surgery at the China-Japan Union Hospital of Jilin University was carried out comprehensively (9). With the approval of the institutional review committee, we retrospectively analyzed the patients with SH. They were in the Department of thyroid surgery of the China- 
Japan Union Hospital of Jilin University from January 2000 to December 2019. To reduce the loss of followup bias, we only included SH in the China-Japan Union Hospital of Jilin University. We reviewed the above 200 patients' medical records to collect detailed information, including clinical history, physical examination, laboratory data, medication status, etc. Among them, 100 patients underwent parathyroidectomy under neurological monitoring (Group A) from 2010-2019, and 100 patients underwent parathyroidectomy without neurological monitoring (Group B) from 2000-2009. We recorded the height, weight, dosage of muscle relaxant, surgery and anesthesia time, perioperative parathyroid hormone (PTH), blood calcium, blood phosphorus, urea, creatinine, ALP, hospital stay, and other data of each patient. Data collection was based on the database of our hospital. Anesthesia records, paper medical records, telephone, and outpatient follow-up were reviewed. We excluded 46 patients who did not receive surgery, data missing, and refused to follow-up.

After that, 62 patients were excluded from the propensity matching score to reduce data bias and confounding variables (Figure 1). The designated anesthesiologist interviewed the remaining 92 patients by telephone or by routine follow-up examination in the outpatient department. There were 46 cases of parathyroidectomy under nerve monitoring (Group A) and 46 points (Group B) without nerve monitoring (Group A), including 56 males and 36 females. The patient was 29-69 years old, weighing 43-77 kg. ASA grade II-III.

\section{Intervention}

After entering the operating room, the venous access was opened, and the lactic acid Ringer's solution was infused with $6-8 \mathrm{~mL} \cdot \mathrm{kg}^{-1} \cdot \mathrm{h}^{-1}$. Routine monitoring of electrocardiogram (ECG), non-invasive blood pressure (BP), blood oxygen saturation $\left(\mathrm{SpO}_{2}\right)$. The built-in gas sampling device is connected to the monitor to routinely monitor the partial pressure of end-expiratory carbon dioxide $\left(\mathrm{ETCO}_{2}\right)$ and MAC values. During surgery, BIS was connected to monitor the patient's conscious state. Before anesthesia induction, pure oxygen was inhaled with a mask for $5 \mathrm{~min}$. After intravenous injection of propofol injection and sufentanil citrate injection $0.4 \mu \mathrm{g} / \mathrm{kg}$. Patients in Group B received 1.5-4 times the standard dose of ED9 cisatracurium, inserted into the regular spring tube and fixed, and combined with inhalation anesthesia to maintain the appropriate depth of anesthesia.

In 2010, the Department of Thyroid Surgery of ChinaJapan Union Hospital of Jilin University introduced and widely carried out the intraoperative monitoring technology of RLN. The Department of Anesthesiology of ChinaJapan Union Hospital of Jilin University summarized a new anesthesia plan for parathyroidectomy patients under nerve monitoring. Based on the previous conventional anesthesia regimen, patients in Group A received preoperative electronic laryngoscopy and tracheal catheter monitoring using RLN (Medtronic Xomed, USA). One times ED95 cisatracurium (one effective dose), conventional doses of propofol and sufentanil were used for induction. The enhanced nerve monitoring catheter was inserted using a visual laryngoscope to ensure that the tracheal catheter's electrode lead was in good contact with the glottis so as not to interfere with the acquisition of nerve EMG signals. After successful intubation, the endotracheal catheter should be appropriately fixed to avoid catheter displacement, bending, and other events. Sevoflurane inhalation was used to maintain anesthesia under and respiratory gas and BIS monitoring, with controlled MAC values ranging from 1.2 to 1.4. During surgery, neuromuscular blocking agents are added according to the patient's body movements or vocal cord movements during intubation. Reasonable use of vasoactive drugs during surgery, strengthening blood gas analysis and monitoring of parathyroid function, and careful extubation after surgery.

\section{Outcomes}

The primary outcome measures were the dosage of muscle relaxant, PTH, serum calcium and phosphorus, urea, creatinine, ALP, etc. The secondary outcome measures were postoperative complications and hospital stay. On November 2, 2017, we completed a particular SH, SS. In the past literature, some SH and SS instances may be found, but SH patients' anesthesia experience undergoing nerve monitoring parathyroidectomy is rarely reported. We desire to collect similar data from all over the world and prepare for a broader international multicenter collection. This is the most extensive, first multinational, international description of the subject in the literature. We believe that there must be tens of thousands of SS patients worldwide, especially in the developing countries of South Asia, Africa, and South America. It is necessary to understand the disease better to give patients appropriate treatment as soon as possible. 


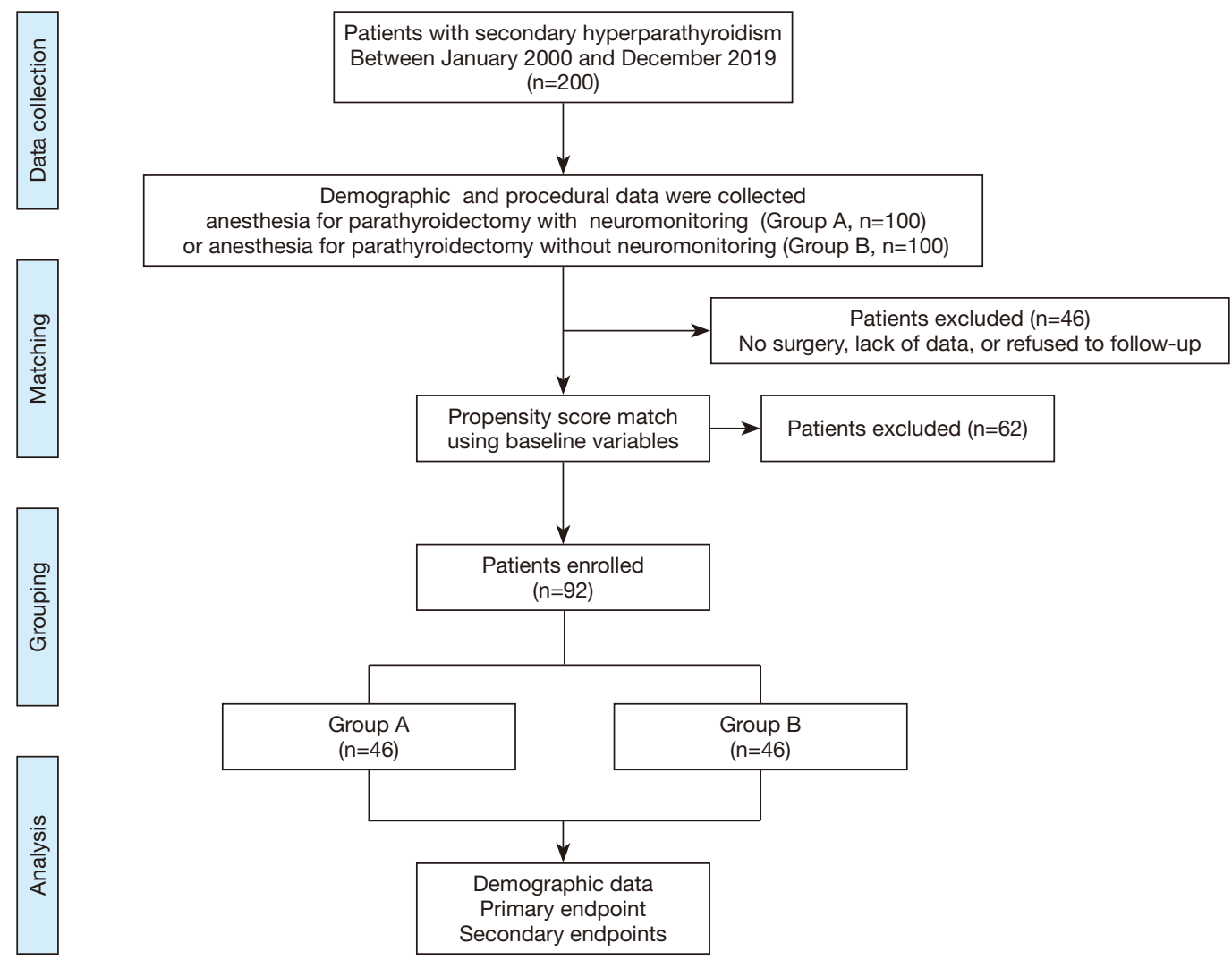

Figure 1 The flow chart.

\section{Statistical analysis}

This study's sample size is calculated based on comparing the advantages of neural monitoring technology in other countries in previous studies. Calculate the sample size $\mathrm{n}=186$. Considering the number of surgeries in our institution and the implementation of intraoperative nerve monitoring technology in 2010, and considering the lack of complete medical records and anesthesia records, we reviewed patients' data from January 2000 to December 2019. This study divided patients undergoing parathyroidectomy into Group A (neuromonitoring group) and Group B (non-neuromonitoring group). SPSS 24.0 software was used for statistical analysis. Graphpad prism 5.0 software is used for drawing. Suppose the measurement data conform to a normal distribution. In that case, it is expressed as mean \pm standard deviation $(\bar{x} \pm \mathrm{s})$, and if it does not work to a normal distribution, it is described as median \pm quartile interval $(\mathrm{m} \pm \mathrm{QR})$. The count data are expressed in constituent ratio or percentage. The independentsample $t$-test was used to fit the normal distribution. The nonparametric rank-sum test was used for the non-normal distribution, and the chi-square test was used for the rate comparison. $\mathrm{P}<0.05$ was statistically significant.

A propensity score-matched analysis was done using a multivariable logistic regression model based on age, sex, bone mineral density, etc. Pairs of patients undergoing neuro-monitored parathyroidectomy versus non-neuromonitored parathyroidectomy were derived using 1:1 greedy nearest neighbor matching within a PS score of 0.02 . This strategy resulted in 46 matched pairs in each group.

\section{Results}

During the study period, a total of 200 patients were selected, of which 46 cases were excluded due to nonsurgery, data loss, refusal to follow-up, and other reasons. The medical records of 154 patients were collected and analyzed. Then PSM was performed, and 92 patients were selected for data analysis. There were 46 patients in Group A and 46 in Group B.

The demographic data, anesthesia time, and surgery 
Table 1 Demographical characteristics and clinical data of the patients

\begin{tabular}{|c|c|c|c|}
\hline Baseline characteristics of the study participants & Group A & Group B & $P$ value \\
\hline Gender (male/female) & $49 / 31$ & $45 / 29$ & 0.955 \\
\hline Age (years) & $47.97 \pm 11.93^{\star}$ & $44.09 \pm 12.33$ & 0.049 \\
\hline Time of parathyroid hormone elevation (months) & $39.80 \pm 23.42^{*}$ & $32.58 \pm 19.42$ & 0.040 \\
\hline Time of renal failure (months) & $89.20 \pm 33.34^{*}$ & $101.01 \pm 40.09$ & 0.048 \\
\hline Bone mineral density (SD) & $-2.31 \pm 1.10^{*}$ & $-2.69 \pm 1.08$ & 0.032 \\
\hline Height (cm) & $166.72 \pm 6.20$ & $165.35 \pm 4.88$ & 0.131 \\
\hline Weight (kg) & $61.78 \pm 8.75$ & $60.06 \pm 7.25$ & 0.188 \\
\hline ASA classification (II-III) & $27 / 53$ & $31 / 43$ & 0.297 \\
\hline \multicolumn{4}{|l|}{ After propensity matching (Group A/Group B =46/46) } \\
\hline Gender (male/female) & $29 / 17$ & $27 / 19$ & 0.669 \\
\hline Age (years) & $45.82 \pm 10.64$ & $44.97 \pm 12.57$ & 0.728 \\
\hline Time of parathyroid hormone elevation (months) & $39.50 \pm 23.17$ & $37.54 \pm 19.91$ & 0.665 \\
\hline Time of bone pain (months) & $20.36 \pm 15.89$ & $21.26 \pm 19.08$ & 0.808 \\
\hline Time of renal failure (months) & $99.13 \pm 29.62$ & $96.17 \pm 42.69$ & 0.702 \\
\hline Bone mineral density (SD) & $-2.60 \pm 1.04$ & $-2.56 \pm 1.16$ & 0.858 \\
\hline
\end{tabular}

Value is mean \pm standard deviation. Group A is the anesthesia for parathyroidectomy with neuromonitoring group; group B is the anesthesia for parathyroidectomy without neuromonitoring group. *, $\mathrm{P}<0.05$. SD, standard deviation; ASA, American Standards Association.

time of the patients are shown in Table 1. There was no significant difference in gender, age, height, weight, PTH rise time, bone pain time, renal failure time, bone mineral density, surgery time, and anesthesia time between the two groups $(\mathrm{P}>0.05)$. Therefore, the muscle relaxant dose of Group A was significantly less than that of Group B $(\mathrm{P}=0.000<0.05)$; the length of hospital stay in Group A was significantly lower than that in Group $\mathrm{B}(\mathrm{P}=0.021<0.05)$. The levels of serum calcium $(\mathrm{P}=0.008)$, serum phosphorus $(\mathrm{P}=0.021)$, urea $(\mathrm{P}=0.002)$ and creatinine $(\mathrm{P}=0.002)$ at $\mathrm{T} 2$ in Group A were lower than those in Group B $(\mathrm{P}<0.05)$ (Table 2). The levels of PTH and ALP had no statistical significance with vital signs such as perioperative body temperature, respiratory rate, heart rate, and blood pressure between the two groups $(\mathrm{P}>0.05)$ (Table 3). The two groups' postoperative complications were observed, and the incidence of postoperative RLN injury, arrhythmia, hypocalcemia, dyspnea, wound hematoma, and other complications were analyzed. RLN injury incidence in Group A was lower than in Group B $(\mathrm{P}=0.041<0.05)$. There 
Table 2 Intraoperative and postoperative outcomes

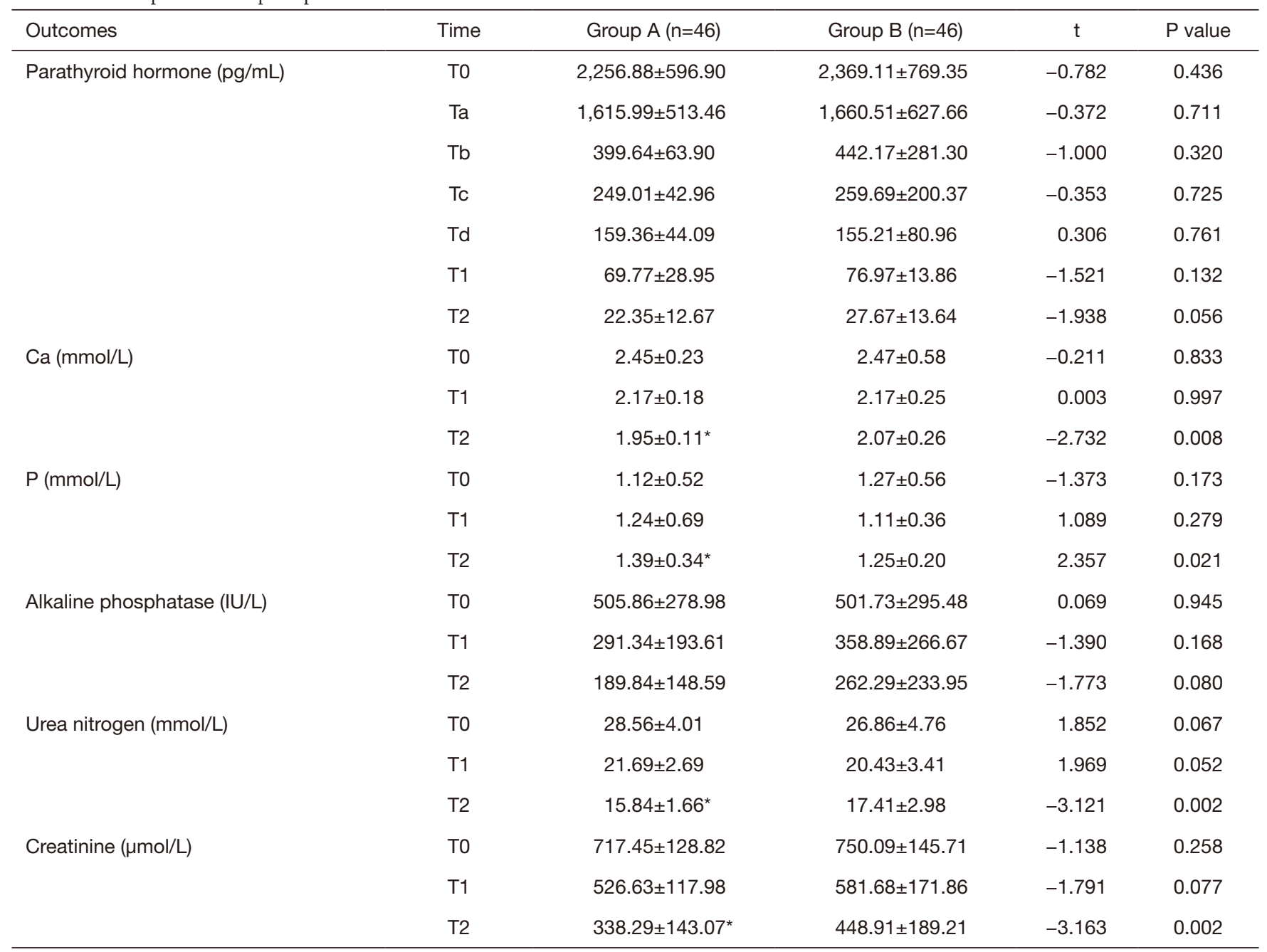

Value is mean \pm standard deviation. Group A is the anesthesia for parathyroidectomy with neuromonitoring group; group $B$ is the anesthesia for parathyroidectomy without neuromonitoring group. * $\mathrm{P}<0.05$.

was no significant difference in the incidence of arrhythmia, hypocalcemia, dyspnea, and wound hematoma between the two groups (Table 4).

\section{Discussion}

One of the main advantages of this study is that we have verified the effectiveness and safety of a new neuromonitoring-based anesthesia experience for hyperparathyroidism. In Table 2, the PTH, alkaline phosphatase (ALP), urea nitrogen, and creatinine in Group A with IONM is lower than that in Group B without IONM at discharge (T2) [PTH $(\mathrm{pg} / \mathrm{mL})$, Group $\mathrm{A}=22.35 \pm 12.67$, vs. Group B =27.67 \pm 13.64 ; ALP (IU/L),
Group A =189.84 \pm 148.59 , vs. Group B =2,262.29 \pm 233.95 ; urea nitrogen $(\mathrm{mmol} / \mathrm{L})$, Group A $=15.84 \pm 1.66$, vs. Group B $=17.41 \pm 2.98$; creatinine $(\mu \mathrm{mol} / \mathrm{L})$, Group $\mathrm{A}=338.29 \pm 143.07$, vs. Group $B=448.91 \pm 189.21]$. In conclusion, $I O N M$ can reduce the PTH and ALP levels and improve renal function in patients with $\mathrm{SH}$ after surgery. There are two technical difficulties in the anesthesia process of parathyroid surgery under nerve monitoring: IONM monitoring the placement of a tracheal tube and the correct use of neuromuscular blocking agents.

First of all, to ensure the neural monitoring loop's smooth surgery, anesthesiologists need to intubate, establish the circle, and maintain the tracheal tube's relative position during the surgery. RLN was used to monitor the tracheal 
Table 3 Changes of vital signs in two groups

\begin{tabular}{lccc}
\hline Vital signs & Time & Group A $(\mathrm{n}=46)$ & Group B $(\mathrm{n}=46)$ \\
\hline Temperature $\left({ }^{\circ} \mathrm{C}\right)$ & T0 & $36.44 \pm 0.26$ & $36.42 \pm 0.22$ \\
Heart (bmp) & T1 & $36.49 \pm 0.27$ & $36.53 \pm 0.21$ \\
Breathe $(\mathrm{bmp})$ & T0 & $76.10 \pm 7.67$ & $79.21 \pm 11.64$ \\
& T1 & $74.10 \pm 6.40$ & $74.58 \pm 7.24$ \\
Blood $(\mathrm{mmHg})$ & T0 & $16.02 \pm 1.29$ & $16.13 \pm 1.16$ \\
& T1 & $15.91 \pm 1.02$ & $16.32 \pm 1.03$ \\
\hline
\end{tabular}

Value is mean \pm standard deviation. Group A is the anesthesia for parathyroidectomy with neuromonitoring group; group B is the anesthesia for parathyroidectomy without neuromonitoring group. Vital signs in two groups have no significant differences $(P>0.05)$.

Table 4 Postoperative complications

\begin{tabular}{|c|c|c|c|}
\hline Postoperative complications & Group A $(n=46)$ & Group B $(n=46)$ & $P$ value \\
\hline Arrhythmology (yes) & $1(2.17 \%)$ & $0(0 \%)$ & 0.315 \\
\hline Hypocalcemia (yes) & $9(19.56 \%)$ & $11(23.91 \%)$ & 0.613 \\
\hline Dyspnea (yes) & $0(0 \%)$ & $1(2.17 \%)$ & 0.315 \\
\hline Hospitalization days (days) & $10.73 \pm 2.34^{*}$ & $11.89 \pm 2.36$ & 0.021 \\
\hline Muscle relaxant dose (mg) & $3.50 \pm 0.62^{*}$ & $5.91 \pm 0.70$ & 0.000 \\
\hline
\end{tabular}

Value is mean \pm standard deviation. Group A is the anesthesia for parathyroidectomy with neuromonitoring group; group B is the anesthesia for parathyroidectomy without neuromonitoring group. ${ }^{*}, \mathrm{P}<0.05$.

tube in our Department (Medtronic Xomed Company, USA). A 40-year-old male patient with bone pain and elevated PTH for 19 months, uremia for 6 years, and hypertension for 7 years. One year ago, the face began to deform. At present, the patient's mandible is protrusive, the bilateral zygomatic eminence, the nose is invaginated, and the lips cannot be closed. Simultaneously, the thoracic skeleton deformity, clubbing fingers in both hands, apparent bone tenderness of the whole body, osteoporosis of the lumbar spine and bilateral hip joints, and height reduction of $10 \mathrm{~cm}$. Preoperative evaluation: ASA III, Mallampati IV, cardiac function III. Before the surgery, we specially asked the Department of Stomatology of our hospital to customize the instruments to protect the patient's teeth in advance. Before intubation, the superficial anesthesia method of tongue root and larynx plus superficial anesthesia with spontaneous breathing reservations was used (17). A visual laryngoscope was first used to understand glottic exposure. Then, 1-fold ED95 cis-atracurium combined with conventional doses of propofol and sufentanil was used for induction, which ensured the smooth intubation process and provided the demand for intraoperative nerve EMG signal monitoring (18). Therefore, a complete airway management plan should be prepared before anesthesia, and maxillofacial and dental protection should be strengthened during intubation. Patients were moved gently after surgery to prevent pathological fracture.

Secondly, patients with hyperparathyroidism may have a history of renal failure for many years (19). For renal failure patients with hypertension control difficulties, anesthesia induction may cause hypotension and other conditions, high blood potassium, high blood calcium 
before parathyroidectomy is easy to cause arrhythmia after resection can cause hypocalcemia, limb convulsion, postoperative laryngeal spasm, a circulatory function is low. We should use vasoactive drugs reasonably, strengthen blood gas analysis and parathyroid function monitoring. During the surgery, BIS was maintained at 40-60, BP 140-150/80-90 mmHg, HR 70-90 bpm, $\mathrm{PETCO}_{2} 35-$ $40 \mathrm{mmHg}$. To limit the liquid input, lactate Ringer solution was used to sustain venous access (no more than $150 \mathrm{~mL} / \mathrm{h}$ ), and no colloid was used for volume expansion. Blood samples were collected at four-time points, a, b, c, and $\mathrm{d}$, at the time points of skin incision, four parathyroid resections, 10 minutes after total resection, and 30 minutes after complete resection. The level of PTH was monitored and finally dropped to more than $85 \%$ after skin incision (20). Serum ions were monitored by blood gas analysis. Before parathyroidectomy, electromyographic signals were measured: $1,419.92 \pm 226.95 \mathrm{uV}$ on the left side and $1,455.37 \pm 208.01 \mathrm{uV}$ on the right side; after parathyroidectomy, the left side was $1,394.00 \pm 223.29 \mathrm{uV}$, and the right side was $1,429.25 \pm 209.38 \mathrm{uV}$, with no significant difference between Group A and Group B. After the surgery, carefully suction pharyngeal secretions, restore the patient's position, stop inhaling anesthetic, MAC value of $0.1 \%$, try to restore spontaneous breathing, ensure that there is no stimulation before the patient automatically wakes up, and carefully extubate after opening eyes to ensure the safety of the patient's airway.

Neurologically monitored parathyroidectomy requires a small quantity of neuromuscular blocking agents (21-23). In our anesthesia protocol, 1-fold ED95 cis-atracurium was used for anesthesia induction. The anesthesia induction process of parathyroidectomy without nerve monitoring was performed with 2-4 times of conventional dose of cisatracurium ED95. Therefore, the muscle relaxant dose of Group A was significantly less than that of Group B. In all successful parathyroidectomy, the PTH should be reduced to more than $85 \%$ before surgery (24). During the surgery, end breath gas, anesthesia depth monitoring, reasonable vasoactive drugs, and other measures to maintain the stability of intraoperative vital signs were used. Therefore, PTH and ALP levels in the two groups were not significantly different from those of the perioperative body temperature, respiratory rate, heart rate, blood pressure, and other vital signs (25). RLN injury is one of the common complications of parathyroidectomy. IONM helps identify and locate the RLN, thus reducing nerve injury incidence (26). For Group A, we used sevoflurane inhalation anesthesia under the monitoring of end breath gas and BIS value, enhanced blood gas analysis and parathyroid function monitoring, timely corrected intraoperative ion imbalance, and reduced perioperative renal injury. Therefore, the serum calcium ion, serum phosphorus ion, urea, and creatinine levels in Group A were significantly lower than those in Group B (Figure 2). Because the incidence of RLN, serum calcium and phosphorus, and urea creatinine levels of patients undergoing parathyroidectomy under nerve monitoring are lower than those of patients undergoing parathyroidectomy without nerve monitoring, this new anesthesia scheme for parathyroidectomy under nerve monitoring can shorten the hospitalization time of patients with $\mathrm{SH}$, which is effective and safe.

This study's disadvantage is that it is a unilateral retrospective study rather than a randomized prospective study. Besides, our findings from a single institution may have limitations. The sample size of this study was small, and the data were collected by the retrospective method. These problems may lead to the deviation of our data and research results. Since the IONM helps identify and locate the RLN, the Surgery time in Group A with IONM should be shorter than Group B without IONM. However, in this study, the surgery time in Group A with IONM is longer than the time in Group B without IONM [Table 1, after propensity matching, surgery time ( $\mathrm{min}$ ) Group A $=170.21 \pm 20.12$, vs. Group B $=162.80 \pm 22.22, \mathrm{P}=0.097]$. This may be due to differences in the length of time that different patients wait for rapid pathological results during surgery or differences in patients' constitutions. Nerve monitoring surgery is the future trend, which can improve the accuracy of surgery (27). In the future, it is hoped that this anesthesia scheme can be extended to more other hospitals in the world, and multicenter research would be carried out to verify its feasibility further.

In a word, parathyroid nerve monitoring technology can reduce the serum calcium and phosphorus ion, creatinine, urea, etc. in patients with hyperparathyroidism, and improve the parathyroid and renal function of patients with hyperparathyroidism, the surgery of parathyroid nerve monitoring technology with complete airway management plan before surgery, anesthesia induction with 1-fold ED95 cis-atracurium, and sevoflurane inhalation anesthesia under BIS monitoring functional prognosis, shorten the length of hospital stay, with the effectiveness and safety. 

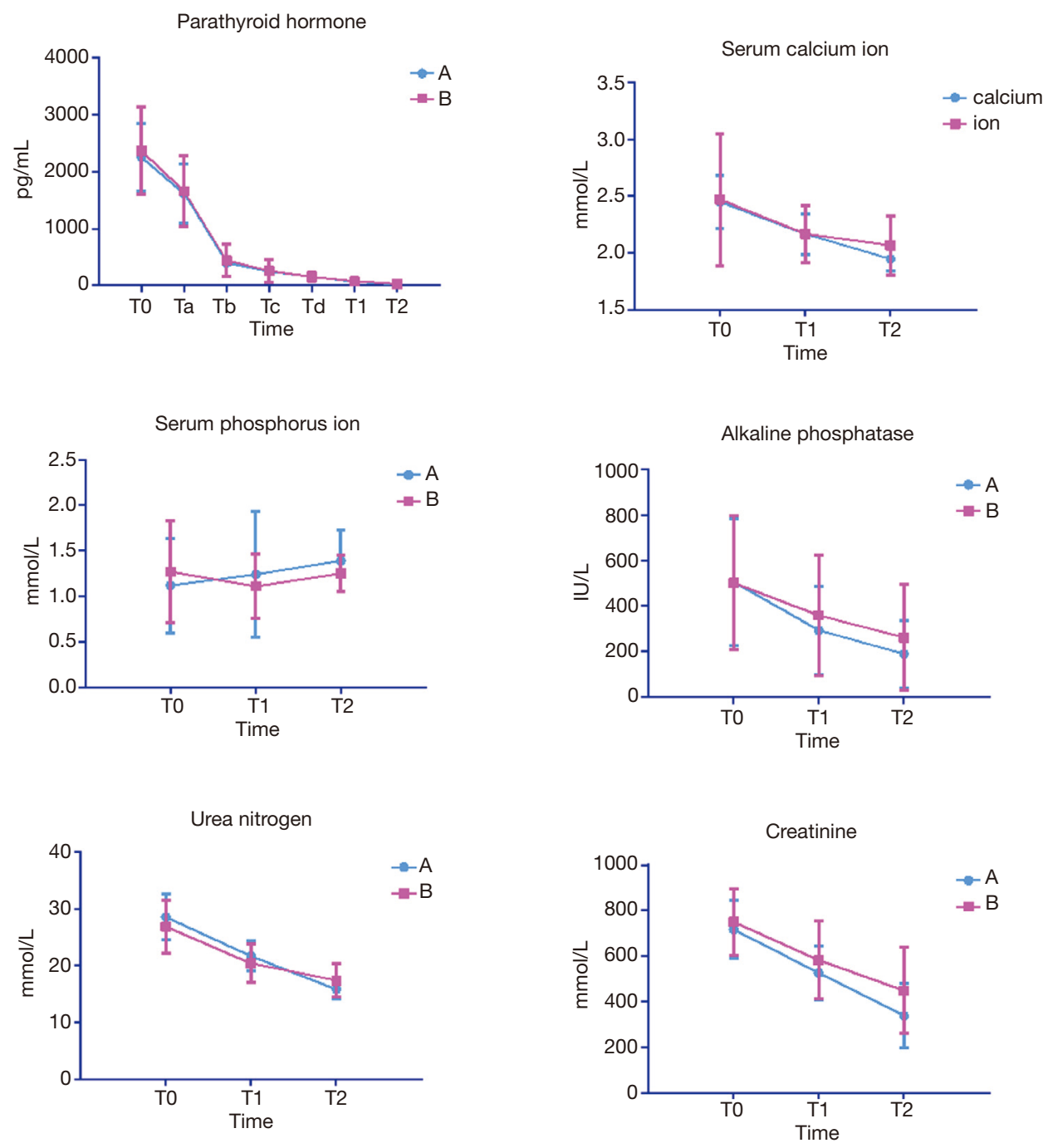

Figure 2 The main results. The midpoints and error bars showed means and SD (standard deviation).

\section{Acknowledgments}

We acknowledge Bing Liu for English language editing.

Funding: None.

\section{Footnote}

Reporting Checklist: The authors have completed the STROBE reporting checklist. Available at http://dx.doi. org/10.21037/gs-21-33

Data Sharing Statement: Available at http://dx.doi. org/10.21037/gs-21-33
Conflicts of Interest: All authors have completed the ICMJE uniform disclosure form (available at http://dx.doi. org/10.21037/gs-21-33). The authors have no conflicts of interest to declare.

Ethical Statement: The authors are accountable for all aspects of the work in ensuring that questions related to the accuracy or integrity of any part of the work are appropriately investigated and resolved. The study was approved by the Ethics Committee of China-Japan Union Hospital of Jilin University (No. 2021-KYLL-030002). The patients have given informed consent. The study was 
conducted in accordance with the Declaration of Helsinki (as revised in 2013).

Open Access Statement: This is an Open Access article distributed in accordance with the Creative Commons Attribution-NonCommercial-NoDerivs 4.0 International License (CC BY-NC-ND 4.0), which permits the noncommercial replication and distribution of the article with the strict proviso that no changes or edits are made and the original work is properly cited (including links to both the formal publication through the relevant DOI and the license). See: https://creativecommons.org/licenses/by-nc-nd/4.0/.

\section{References}

1. Mizobuchi M, Ogata H, Koiwa F. Secondary Hyperparathyroidism: Pathogenesis and Latest Treatment. Ther Apher Dial 2019;23:309-18.

2. Komaba H, Kakuta T, Fukagawa M. Management of secondary hyperparathyroidism: how and why? Clin Exp Nephrol 2017;21:37-45.

3. Alfieri C, Regalia A, Zanoni F, et al. The Importance of Adherence in the Treatment of Secondary Hyperparathyroidism. Blood Purif 2019;47:37-44.

4. Rubio-Manzanares Dorado M, Pino Díaz V, Fontillón Alberdi M, et al. Sagliker Syndrome. Cir Esp (Engl Ed) 2020,98:414-6.

5. Shakeri S, Zarehparvar Moghadam S, et al. Sagliker Syndrome in a Patient with Secondary Hyperparathyroidism and Chronic Renal Insufficiency: A Case Report. Asia Ocean J Nucl Med Biol 2018;6:167-70.

6. Panezai MA, Ahmed S, Colbert GB. Sagliker syndrome in a patient with end-stage renal disease with secondary hyperparathyroidism. Proc (Bayl Univ Med Cent) 2019;32:624-6.

7. Tunç E, Demirhan O, Sağliker Y, et al. Chromosomal findings and sequence analysis of target exons of calciumsensingreceptor (CaSR) gene in patients with Sagliker syndrome. Turk J Med Sci 2017;47:13-21.

8. Lau WL, Obi Y, Kalantar-Zadeh K. Parathyroidectomy in the Management of Secondary Hyperparathyroidism. Clin J Am Soc Nephrol 2018;13:952-61.

9. Zhang D, Pino A, Caruso E, et al. Neural monitoring in thyroid surgery is here to stay. Gland Surg 2020;9:S43-S46.

10. Medniuk A, Bareisiene D, Ahmad I. Nerve integrity monitor tubes for thyroid surgery. Anaesthesia 2014;69:287-8.
11. Marshall SD, Serpell J. Failed Vocalis Muscle Monitoring During Thyroid Surgery Resulting From Residual Muscle Relaxation. A A Case Rep 2017;9:158.

12. Wu CW, Huang TY, Chen HC, et al. Intra-Operative Neural Monitoring of Thyroid Surgery in a Porcine Model. J Vis Exp 2019;11:144.

13. Gardner IH, Doherty GM, McAneny D. Intraoperative nerve monitoring during thyroid surgery. Curr Opin Endocrinol Diabetes Obes 2016;23:394-9.

14. Pachuski J, Vaida S, Donahue K, et al. Effect of laryngotracheal topical anesthesia on recurrent laryngeal nerve monitoring during thyroid Surgery. J Clin Anesth 2016;29:10-3.

15. Chen P, Liang F, Li LY, et al. Complications and adverse effects associated with intraoperative nerve monitoring during thyroid surgery under general anesthesia. Cell Biochem Biophys 2015;71:1029-33.

16. Brozović G, Oršolić N, Rozgaj R, et al. Sevoflurane and isoflurane genotoxicity in kidney cells of mice. Arh Hig Rada Toksikol 2017;68:228-35.

17. Bjurström MF, Bodelsson M, Sturesson LW. The Difficult Airway Trolley: A Narrative Review and Practical Guide. Anesthesiol Res Pract 2019;2019:6780254.

18. Terris DJ, Anderson SK, Watts TL, et al. Laryngeal nerve monitoring and minimally invasive thyroid surgery: complementary technologies. Arch Otolaryngol Head Neck Surg 2007;133:1254-7.

19. Komaba H, Fukagawa M. Secondary Hyperparathyroidism and Protein-Energy Wasting in End-Stage Renal Disease. Ther Apher Dial 2018;22:246-50.

20. Cinamon U, Gavish D, Ovnat Tamir S, et al. Effect of general anesthesia and intubation on parathyroid levels in normal patients and those with hyperparathyroidism. Head Neck 2018;40:555-60.

21. Seo KH, Kim KM, Lee SK, et al. Comparative Analysis of Phase Lag Entropy and Bispectral Index as Anesthetic Depth Indicators in Patients Undergoing Thyroid Surgery with Nerve Integrity Monitoring. J Korean Med Sci 2019;34:e151.

22. Randolph GW, Dralle H, International Intraoperative Monitoring Study Group, et al. Electrophysiologic recurrent laryngeal nerve monitoring during thyroid and parathyroid surgery: international standards guideline statement. Laryngoscope 2011;121:S1-16.

23. Rao SD. Epidemiology of parathyroid disorders. Best Pract Res Clin Endocrinol Metab 2018;32:773-80.

24. Galassi A, Ciceri P, Fasulo E, et al. Management of secondary hyperparathyroidism in chronic kidney disease: 
a focus on the elderly. Drugs Aging 2019;36:885-95.

25. Randolph GW, Kamani D. Intraoperative neural monitoring in thyroid cancer surgery. Langenbecks Arch Surg 2014;399:199-207.

26. Gür EO, Haciyanli M, Karaisli S, et al. Intraoperative nerve monitoring during thyroidectomy: evaluation of signal loss, prognostic value and surgical strategy. Ann R
Coll Surg Engl 2019;101:589-95.

27. Henry BM, Graves MJ, Vikse J, et al. The current state of intermittent intraoperative neural monitoring for prevention of recurrent laryngeal nerve injury during thyroidectomy: a PRISMA-compliant systematic review of overlapping meta-analyses. Langenbecks Arch Surg 2017;402:663-73.
Cite this article as: Wang D, Li L, Zhang C, Liang F, Chen P. A new anesthesia scheme for parathyroidectomy under neuromonitoring: a retrospective cohort study. Gland Surg 2021;10(5):1576-1586. doi: 10.21037/gs-21-33 